\title{
NOVO MODELO DE ENERGIA DE GIBBS PARA O ESPINÉLIO AL $\mathrm{MNO}_{4}$
}

Rogério Navarro Correia de Siqueira ${ }^{\prime}$ Roberto Ribeiro de Avillez ${ }^{2}$ Angelo Márcio de Souza Gomez ${ }^{3}$

\section{Resumo}

Medidas de capacidade calorífica à pressão constante na faixa entre 2 e $873 \mathrm{~K}$ permitem a construção de um novo modelo de energia de Gibbs molar para o espinélio $\mathrm{Al}_{2} \mathrm{MnO}_{4}$ :

$$
G=-2014360.336+2265.652 T-295.448 T \ln T-157.312 .10^{2} \sqrt{T}-\frac{88.5445 .10^{5}}{T}+\frac{62.5042 .10^{7}}{T^{2}}
$$

O modelo é empregado no acesso do diagrama de fases do sistema $\mathrm{Al}_{2} \mathrm{O}_{3}-\mathrm{MnO}$ e permite uma melhor descrição dos dados termodinâmicos disponíveis na literatura em comparação com o modelo já existente no banco SSUB3.

Palavras-chave: Energia de Gibbs; $\mathrm{Al}_{2} \mathrm{MnO}_{4}$; Capacidade calorífica a pressão constante.

\section{A NOVEL GIBBS ENERGY MODEL FOR THE SPINEL AL $\mathrm{MNO}_{4}$}

\section{Abstract}

Measurements of molar heat capacity at constant pressure in the range between 2 and $873 \mathrm{~K}$ enable the construction of a new molar Gibbs energy model for the spinel $\mathrm{Al}_{2} \mathrm{MnO}_{4}$ :

$$
G=-2014360.336+2265.652 T-295.448 T \ln T-157.312 .10^{2} \sqrt{T}-\frac{88.5445 .10^{5}}{T}+\frac{62.5042 .10^{7}}{T^{2}}
$$

The new model is applied in the assessment of the system $\mathrm{Al}_{2} \mathrm{O}_{3}-\mathrm{MnO}$, and results in a better overall description of the available thermodynamic data in comparison with the results obtained with the model already implemented in the SSUB3 database.

Key words: Gibbs energy; $\mathrm{Al}_{2} \mathrm{MnO}_{4}$; Heat capacity at constant pressure.

\section{O SISTEMA AL $\mathrm{O}_{3}-\mathrm{MNO}$}

$\mathrm{O}$ sistema $\mathrm{Al}_{2} \mathrm{O}_{3}-\mathrm{MnO}$ vem despertando a atenção da comunidade científica há bastante tempo. A primeira investigação diz respeito à pesquisa conduzida por Olsen e Heyner, ${ }^{(1)}$ que obtiveram pontos da linha liquidus de misturas dos óxidos $\mathrm{Al}_{2} \mathrm{O}_{3}, \mathrm{MnO}$, e $\mathrm{FeO}$, equilibradas em cadinhos de alumina. As amostras eram aquecidas até uma dada temperatura, em que permaneciam até o estabelecimento do equilíbrio, sendo, em seguida, resfriadas rapidamente. A microestrutura era analisada via microscopia óptica. Olsen e Heynert ${ }^{(1)}$ estudam misturas com fração mássica de $\mathrm{FeO}$ igual ou superior $20 \%$; a extrapolação dos dados até a condição em que a fração mássica de $\mathrm{FeO}$ é igual a zero e permite a obtenção de estimativas para pontos característicos da linha liquidus do mencionado sistema. Olsen e Heynert ${ }^{(1)}$ detectam a presença de duas reações invariantes: uma na região rica no componente $\mathrm{Al}_{2} \mathrm{O}_{3}(2.056 \pm 15 \mathrm{~K})$ e outra na região rica em $\mathrm{MnO}$ ( $1.793 \pm 15 \mathrm{~K})$. Os dados sugerem que o óxido duplo $\mathrm{Al}_{2} \mathrm{MnO}_{4}$ se funde de forma incongruente em uma reação peritética em torno de $2.056 \pm 15 \mathrm{~K}$, e forma um eutético na região rica em $\mathrm{MnO}$, de isoterma em torno de $\mathrm{I} .793 \pm \mathrm{I} 5 \mathrm{~K}$.

Posteriormente, Novokahatski et al. ${ }^{(2)}$ estudaram a fusão dos componentes $\mathrm{Al}_{2} \mathrm{O}_{3}, \mathrm{MnO}$ e $\mathrm{Al}_{2} \mathrm{MnO}_{4}$ construindo cones pirométricos; os cones eram aquecidos em atmosfera constituída por argônio e hidrogênio e a defor-

\footnotetext{
' Engenheiro químico, D.C. e pós doutorando da Universidade Católica do Rio de Janeiro, Departamento de Engenharia de Materiais, PUC- Rio, Rua Marquês de São Vicente, Gávea, Cep 2245 I-900, Rio de Janeiro, RJ, Brasil. E-mail: rogerioncs@gmail.com

${ }^{2}$ Engenheiro metaúrico, PHD e professor da Universidade Católica do Rio de Janeiro, Departamento de Engenharia de Materiais, PUC-Rio, Rua Marquês de São Vicente, Gávea, Cep 2245 I-900, Rio de Janeiro, RJ, Brasil. E-mail: avillez@puc-rio.br

${ }^{3}$ Físico, PHD, processor da Universidade Federal do Estado do Rio de Janeiro, Instituto de Física, Universidade Federal do Rio de Janeiro - UFRJ, Centro de Tecnologia, Bloco A, Cidade Universitária, Cep 2194I-909, Rio de Janeiro, RJ, Brasil. E-mail: amgomes@if.ufrj.br.
} 
mação permanente do cone indicava $\circ$ início da fusão. Novokahatski et al. ${ }^{(2)}$ concluem que o óxido $\mathrm{Al}_{2} \mathrm{MnO}_{4}$ se funde de maneira congruente em 2.123 $\pm 15 \mathrm{~K}$. $\mathrm{O}$ autor emprega a mesma técnica para determinar as temperaturas invariantes presentes no sistema e verifica que o óxido $\mathrm{Al}_{2} \mathrm{MnO}_{4}$ participa de dois eutéticos: um no lado rico em $\mathrm{MnO}$ de isoterma definida por $1.793 \pm 15 \mathrm{~K}$ e outro no lado rico em $\mathrm{Al}_{2} \mathrm{O}_{3}$, de magnitude igual a $1.993 \pm 15 \mathrm{~K}$.

Alguns anos depois, Jacob ${ }^{(3)}$ realizou uma série de experimentos no pseudo-binário $\mathrm{Al}_{2} \mathrm{O}_{3}-\mathrm{MnO}$, visando tanto a determinação de pontos da linha líquidus do sistema, quanto para medidas de valores de atividade química do óxido $\mathrm{MnO}$. Nos experimentos para o estudo do diagrama de fases, Jacob ${ }^{(3)}$ utilizou inicialmente um forno com atmosfera evacuada, onde mediu as temperaturas de fusão dos óxidos puros, bem como também os pontos eutéticos em ambos os lados do diagrama. As amostras eram mantidas por trinta minutos em cada temperatura, sendo então rapidamente resfriadas mediante contato com o ar atmosférico. As composições iniciais de cada mistura foram determinadas via difração de raios $X$. A composição do líquido nos pontos eutéticos foi avaliada mediante análise pela técnica de microscopia eletrônica de varredura, a partir da aquisição de espectros de dispersão de raios $X$ (EDS). Pontos da linha liquidus na região rica em $\mathrm{MnO}$ foram obtidos em uma série de outros experimentos, mediante $\circ$ equilíbrio de misturas contendo $\mathrm{MnO}$ e $\mathrm{Al}_{2} \mathrm{MnO}_{4}$ em cadinhos de platina. A atmosfera era constituída por uma mistura de argônio e oxigênio; nesses experimentos, a pressão parcial de oxigênio variou na faixa entre $10^{-6} \mathrm{~atm}$ e $10^{-8} \mathrm{~atm}$. $O$ controle da pressão parcial de oxigênio inibe a contaminação da fase espinélio por espécies $\mathrm{Mn}^{+3}$, como também a redução do manganês a manganês metálico, que poderia difundir para o cadinho. As misturas eram resfriadas com nitrogênio líquido e a composição das fases determinada via EDS. $O$ autor detectou a ocorrência de solubilidade parcial do $\mathrm{Al}_{2} \mathrm{O}_{3}$ na estrutura do $\mathrm{MnO}$ no experimento realizado em I.973K. $\mathrm{Jacob}^{(3)}$ estuda, ainda, o equilíbrio em uma mistura de $\mathrm{MnO}$ e manganês metálico em um cadinho selado de alumina em I.823K e 1.973K. Após três horas o cadinho foi seccionado e a secção analisada em microscópico eletrônico de varredura. Foi possível detectar a formação de cristais do óxido $\mathrm{Al}_{2} \mathrm{MnO}_{4}$ na região próxima à parede do cadinho. Análises por EDS, conduzidas em amostras oriundas do equilíbrio em I.973K, evidenciaram reduzida solubilidade do $\mathrm{MnO}$ na estrutura do $\mathrm{Al}_{2} \mathrm{O}_{3}$. Adicionalmente, a estrutura do óxido $\mathrm{Al}_{2} \mathrm{MnO}_{4}$ apresenta deficiência de manganês, tanto em I.823K quanto em I.973K, ou seja, comporta-se como uma fase não estequiométrica no equilíbrio estabelecido nessas temperaturas. A temperatura de fusão congruente do óxido $\mathrm{Al}_{2} \mathrm{MnO}_{4}(2.114 \pm 15 \mathrm{~K})$ bem como as isotermas eutéticas $(2.030 \pm 15 \mathrm{~K}$ e $1802 \pm 15 \mathrm{~K})$, medidas por Jacob $^{(3)}$, apresentam concordância com os valores reportados vinte anos antes por Novokahatski et al. ${ }^{(2)}$.
Dados de atividade química do $\mathrm{MnO}$ na fase escória são determinados por Jacob(3) mediante $\circ$ equilíbrio de misturas dos óxidos $\mathrm{Al}_{2} \mathrm{O}_{3}, \mathrm{Al}_{2} \mathrm{MnO}_{4}$ e $\mathrm{MnO}$ em contato com um fio de platina em atmosfera controlada. A concentração de oxigênio na atmosfera foi fixada mediante a injeção de uma mistura composta por $\mathrm{CO}$ e $\mathrm{CO}_{2}$. Após o equilíbrio, que segundo o autor era alcançado após oito horas, pode ser aferida a fração atômica de manganês na platina. Com base nesse valor, pode-se calcular a atividade química do $\mathrm{MnO}$ na fase escória. ${ }^{(3)} \mathrm{A}$ técnica foi utilizada para a obtenção de valores de atividade em $1.873 \mathrm{~K}$ e 1.923K. Os dados em 1923K mostram-se ligeiramente distintos dos obtidos anos antes por Sharma e Richardson ${ }^{(4)}$ mediante a aplicação da mesma técnica.

Jung et al..$^{(5)}$ acessaram as propriedades termodinâmicas do sistema $\mathrm{Al}_{2} \mathrm{O}_{3}-\mathrm{MnO}$, e argumentam que a proposta de que o óxido $\mathrm{Al}_{2} \mathrm{MnO}_{4}$ se funde de forma congruente não permite um ajuste satisfatório da totalidade dos dados termodinâmicos publicados para este sistema, indo de encontro aos resultados de Novokahatski et al. ${ }^{(2)}$ e Jacob(3). Convém comentar, no entanto, que não existem dados termodinâmicos publicados na literatura que permitam uma precisa avaliação da energia de Gibbs da fase espinélio, ou seja, a dificuldade encontrada por Jung et al..$^{(5)}$ pode ser oriunda não da inconsistência presente nos dados obtidos pelos mencionados autores, mas sim pela utilização de um modelo de energia de Gibbs do óxido $\mathrm{Al}_{2} \mathrm{MnO}_{4}$ inconsistente com a realidade.

O presente trabalho apresenta como objetivo a proposição de um novo modelo de energia de Gibbs para - espinélio $\mathrm{Al}_{2} \mathrm{MnO}_{4}$ em sua forma estequiométrica, cuja construção fundamenta-se em dados experimentais de capacidade térmica à pressão constante na faixa de $2 \mathrm{~K}$ a $873 \mathrm{~K}$, obtidos recentemente por Navarro.(6) $\mathrm{O}$ novo modelo é comparado com o modelo existente no banco SSUB3 ${ }^{(7)}$ no que diz respeito ao acesso das informações termodinâmicas disponíveis na literatura para o pseudobinário $\mathrm{Al}_{2} \mathrm{O}_{3}-\mathrm{MnO}$. Todos os cálculos foram realizados empregando-se o software Thermocalc.

\section{AVALIAÇÃO TERMODINÂMICA PRELIMINAR}

Foi realizada uma avaliação preliminar do sistema $\mathrm{Al}_{2} \mathrm{O}_{3}-\mathrm{MnO}$ com o intuito de se comparar as duas hipóteses atualmente existentes para a fusão do óxido $\mathrm{Al}_{2} \mathrm{MnO}_{4}$. O procedimento seguido é consistente com a metodologia Calphad. ${ }^{(8)}$ Todos os óxidos puros $\left(\mathrm{Al}_{2} \mathrm{O}_{3}\right.$, $\mathrm{MnO}$, e $\mathrm{Al}_{2} \mathrm{MnO}_{4}$ ) são tratados como estequiométricos, empregando-se as informaçóes contidas no banco de dados SSUB3. ${ }^{(7)}$ A energia de Gibbs da fase escória é calculada com o modelo de Kapoor-Frohberg-Gaye. ${ }^{(9)}$

Em ambos os casos os dados da curva associada à curva referente à linha liquidus na região rica em $\mathrm{MnO}$ podem ser descritos de forma quantitativa (Figuras I e 2). Nestas apenas os dados representados em azul são incluídos no ajuste. 


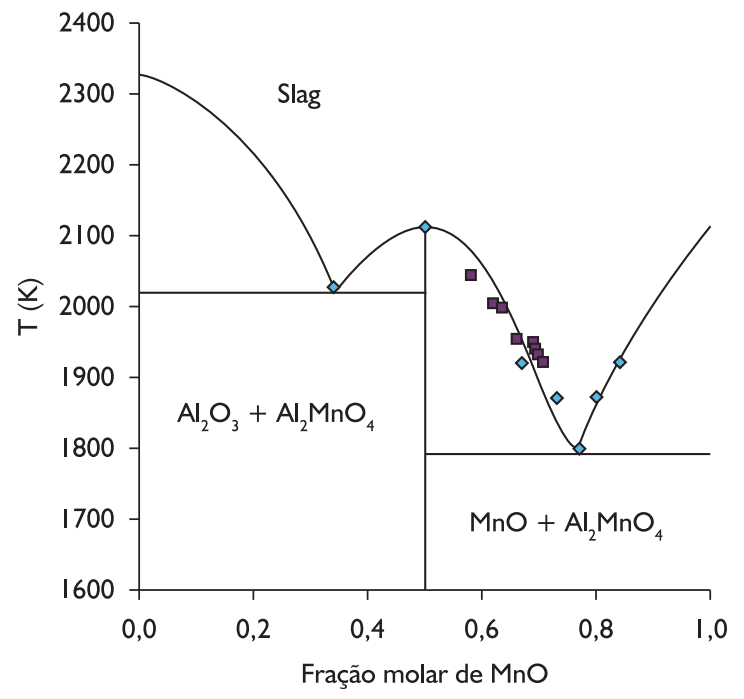

$\diamond$ Jacob (3) $\square$ Olsen e heynert (I)

Figura I. Diagrama de equilíbrio de fases hipotético para o sistema $\mathrm{Al}_{2} \mathrm{O}_{3}-\mathrm{MnO}$ - fusão congruente do óxido $\mathrm{Al}_{2} \mathrm{MnO}_{4}$.

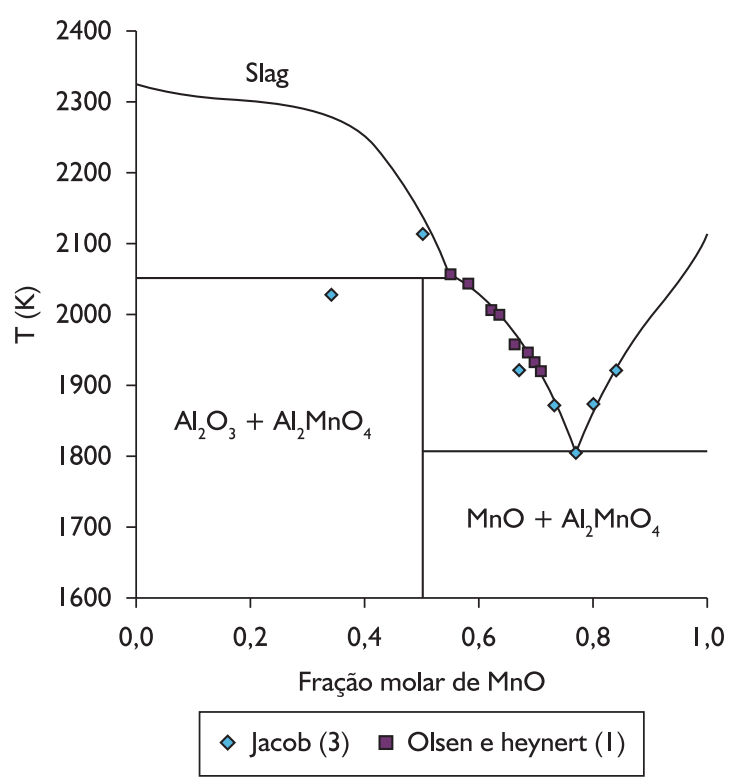

Figura 2. Diagrama de equilíbrio de fases hipotético para o sistema $\mathrm{Al}_{2} \mathrm{O}_{3}-\mathrm{MnO}$ - decomposição peritética do óxido $\mathrm{Al}_{2} \mathrm{MnO}_{4}$.

A linha liquidus na região rica em $\mathrm{Al}_{2} \mathrm{O}_{3}$, calculada com os parâmetros do ajuste considerando a reação peritética (Figura 2), apresenta uma curvatura indicativa de uma reação tipo decomposição espinodal (ponto de inflexão). Não existem dados na literatura que suportem este fenômeno para o presente sistema. Adicionalmente, no que diz respeito aos dados de atividade química, a hipótese de que o óxido $\mathrm{Al}_{2} \mathrm{MnO}_{4}$ se decompõe através de uma reação peritética impede a descrição satisfatória dos patamares de isoatividade em I.923K e I.873K (Figuras 3 e 4).
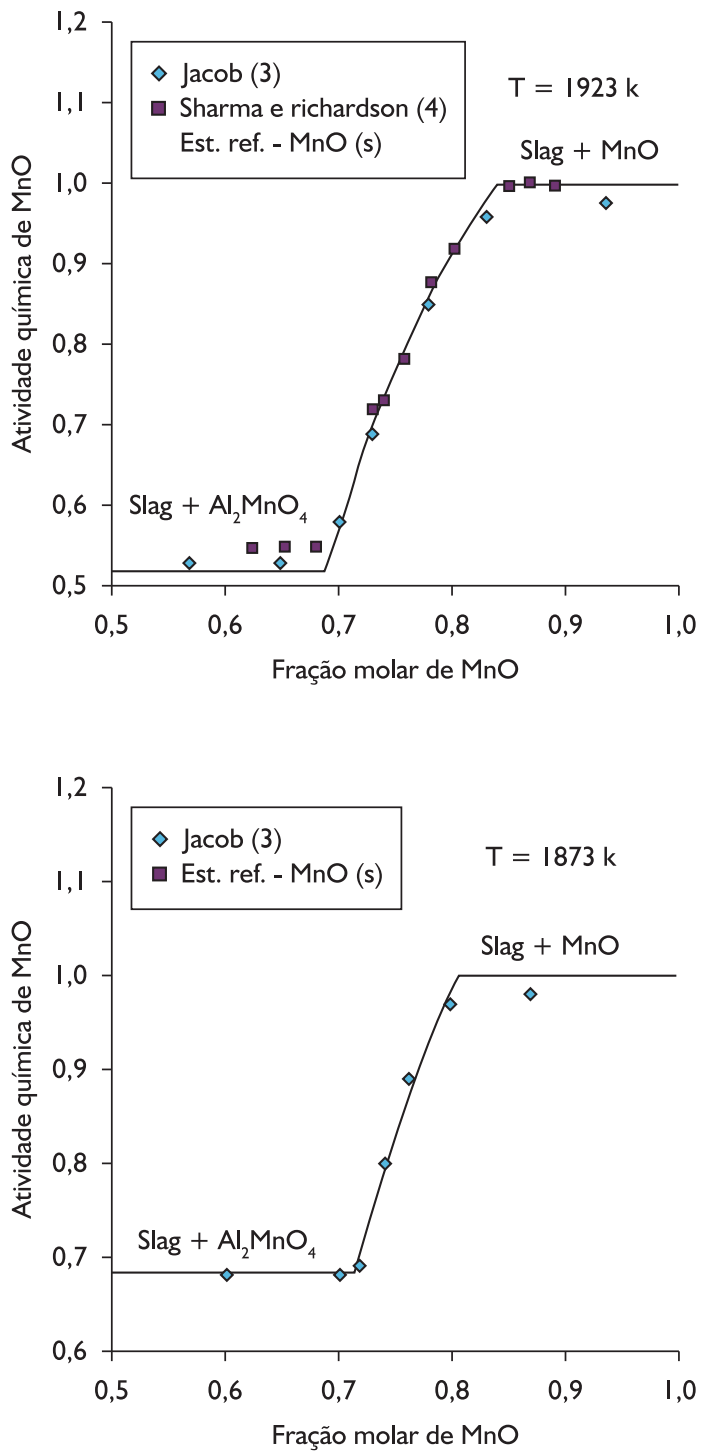

Figura 3. Atividade química do $\mathrm{MnO}$ em I.923K e I.873K - fusão congruente do óxido $\mathrm{Al}_{2} \mathrm{MnO}_{4}$.

Por outro lado, de forma consistente com os comentários de Jung et al. ${ }^{(5)}$, os parâmetros estimados para a fase escória, bem como também o parâmetro de correção da energia de Gibbs do espinélio $\mathrm{Al}_{2} \mathrm{MnO}_{4}$ apresentam magnitudes significativamente mais expressivas no ajuste com a fusão congruente (Tabela I). Tal comparação não permite por si só descartar a hipótese de fusão congruente, pois o modelo de energia de Gibbs do óxido $\mathrm{Al}_{2} \mathrm{MnO}_{4}$ proveniente do SSUB3 não se apresenta fundamentado em dados termodinâmicos reais da fase em questão.

\section{ENERGIA DE GIBBS DO ESPINÉLIO AL $\mathrm{MNO}_{4}$}

A dependência térmica da energia de Gibbs molar de um óxido estequiométrico qualquer pode ser modelada para temperaturas acima de $298,15 \mathrm{~K}$, uma vez conhecendo-se a entropia molar e entalpia de formação 

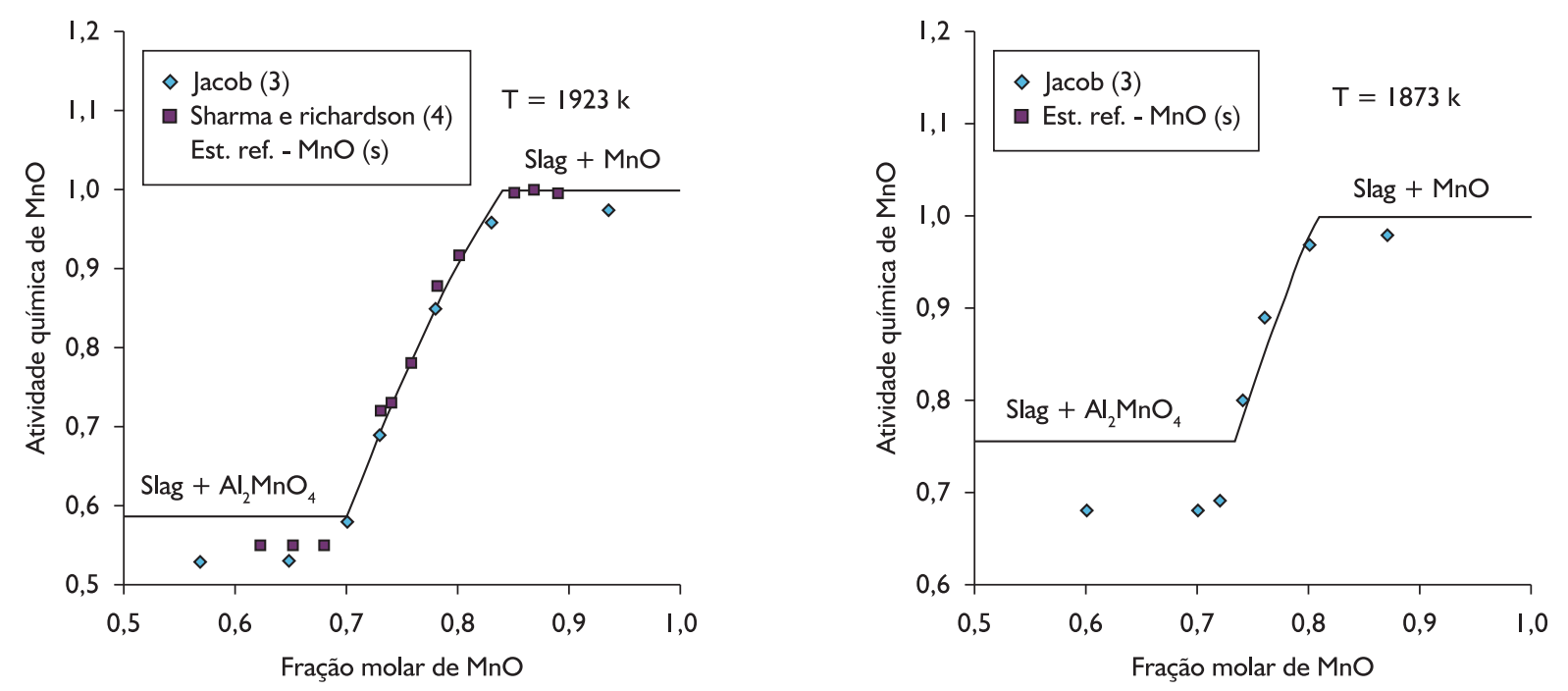

Figura 4. Atividade química do $\mathrm{MnO}$ em I.923K e I.873K - decomposição peritética do óxido $\mathrm{Al}_{2} \mathrm{MnO}_{4}$.

Tabela I. Parâmetros estimados no ajuste preliminar

\begin{tabular}{cccc}
\hline$E_{\mathrm{Mn}, \mathbf{A l}}$ & $\mathbf{W}_{\mathbf{M n}, \mathbf{A l}}$ & Correção $\mathbf{G}_{\mathbf{A l}_{2} \mathbf{M n O}_{4}}$ & Ajuste \\
\hline$-26155+24832 . X_{\mathrm{Al}^{+3}}$ & $-48369-39497 . X_{\mathrm{Al}^{+3}}$ & -58325 & Fusão congruente \\
$-6752+433 \mid 3 . \mathrm{X}_{\mathrm{Al}^{+3}}$ & $-13449-3208 I . \mathrm{X}_{\mathrm{Al}^{+3}}$ & $-554 \mid+0.00978 T$ & Peritético \\
\hline
\end{tabular}

a $298,15 \mathrm{~K}$ na pressão de interesse, e um modelo para a capacidade térmica nesta mesma pressão, que funcione de $298,15 \mathrm{~K}$ até a temperatura onde se deseja conhecer as propriedades do sistema. Fixando-se a pressão em uma atmosfera, tem-se:

$$
\mathrm{G}^{0}=\mathrm{H}_{298}^{0}-\mathrm{TS}_{298}^{0}+\int_{298.15}^{\mathrm{T}} \mathrm{C}_{\mathrm{p}}^{0} \mathrm{dT}-\mathrm{T} \int_{298.15}^{\mathrm{T}}\left(\frac{\mathrm{C}_{\mathrm{P}}^{0}}{\mathrm{~T}}\right) \mathrm{dT}
$$

Recentemente, Navarro(6) apresenta dados de capacidade calorífica $\left(C_{\mathrm{p}}\right)$ do óxido $\mathrm{Al}_{2} \mathrm{MnO}_{4}$ para I atm, na faixa entre $2 \mathrm{~K}$ e $873 \mathrm{~K}$. Os valores obtidos na faixa entre $2 \mathrm{~K}$ e $300 \mathrm{~K}$ foram utilizados para o cálculo da entropia molar a $298,15 \mathrm{~K}$. Os valores entre $300 \mathrm{~K}$ e $873 \mathrm{~K}$ foram modelados pela função proposta por Berman e Brown, ${ }^{(10)}$ representada na Equação 2:

$$
C_{p}=k_{0}+k_{1} T^{-0.5}+k_{2} T^{-2}+k_{3} T^{-3}
$$

Os parâmetros $k_{0}, k_{1}, k_{2}, e k_{3}$ são ajustados aos dados experimentais característicos de cada fase. A função (2) apresenta $O$ atrativo de resultar em extrapolações satisfatórias em temperaturas elevadas. ${ }^{(10)}$ Isto é importante, pois o modelo de interesse deve ser válido até a temperatura de fusão congruente do óxido (2.214K). Empregando-se os dados experimentais sugeridos por $\mathrm{Navarro}^{(6)}$, em conjunto com o valor de entalpia de formação proveniente do banco SSUB3, chega-se à seguinte equação para o cálculo da energia de Gibbs do óxido $\mathrm{Al}_{2} \mathrm{MnO}_{4}$ :

$$
\begin{aligned}
G= & 20 \mid 4360.336+2265.652 T 295.448 T \ln T \\
& \mid 57.312 .10^{2} \sqrt{T} \frac{88.5445 \cdot 10^{5}}{T}+\frac{62.5042 .10^{7}}{T^{2}}
\end{aligned}
$$

A função (3) é termodinamicamente consistente, uma vez que seu gráfico apresenta natureza côncava para toda a faixa de temperatura. $O$ novo modelo mostra-se idêntico ao modelo contido no banco SSUB3 em temperaturas próximas a 298,15K; porém, em temperaturas elevadas, podem ser notadas discrepâncias significativas (Figura 5).

\section{REAVALIAÇÃO TERMODINÂMICA DO SISTEMA AL $\mathrm{O}_{3}$ - MNO}

Empregando-se os mesmos dados experimentais utilizados no ajuste descrito no tópico (2) realizou-se novamente $\circ$ acesso termodinâmico do sistema $\mathrm{Al}_{2} \mathrm{O}_{3}-\mathrm{MnO}$, substituindo-se $\circ$ modelo do SSUB3 ${ }^{(7)}$ para a energia de Gibbs da fase espinélio pelo modelo aqui proposto. Percebe-se claramente que todos os dados podem ser descritos de forma quantitativa (Figura 6). No entanto, podem ser observados desvios significativos nos pontos eutéticos, conforme indicam os dados da Tabela 2.

De acordo com Jacob,,$^{(3)}$ o equilíbrio nas isotermas eutéticas deve ser fortemente influenciado pelo fato do óxido $\mathrm{Al}_{2} \mathrm{MnO}_{4}$ apresentar natureza não estequiométrica em temperaturas acima de $1.700 \mathrm{~K}$. de acordo com o autor, cátions $\mathrm{Mn}^{+2}$ e $\mathrm{Al}^{+3}$ podem trocar de posição 


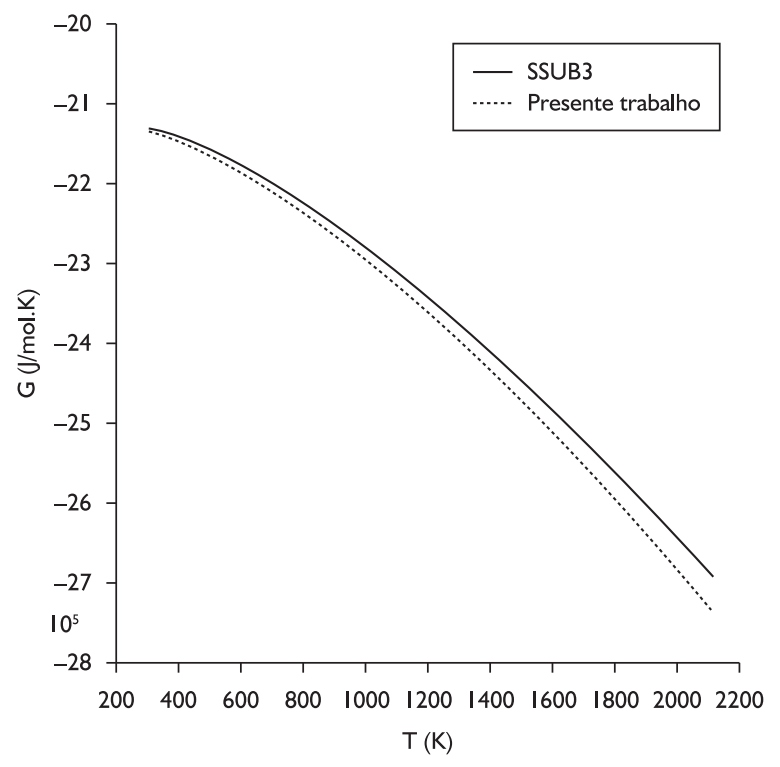

Figura 5. Modelos de energia de Gibbs para o óxido $\mathrm{Al}_{2} \mathrm{MnO}_{4}$.

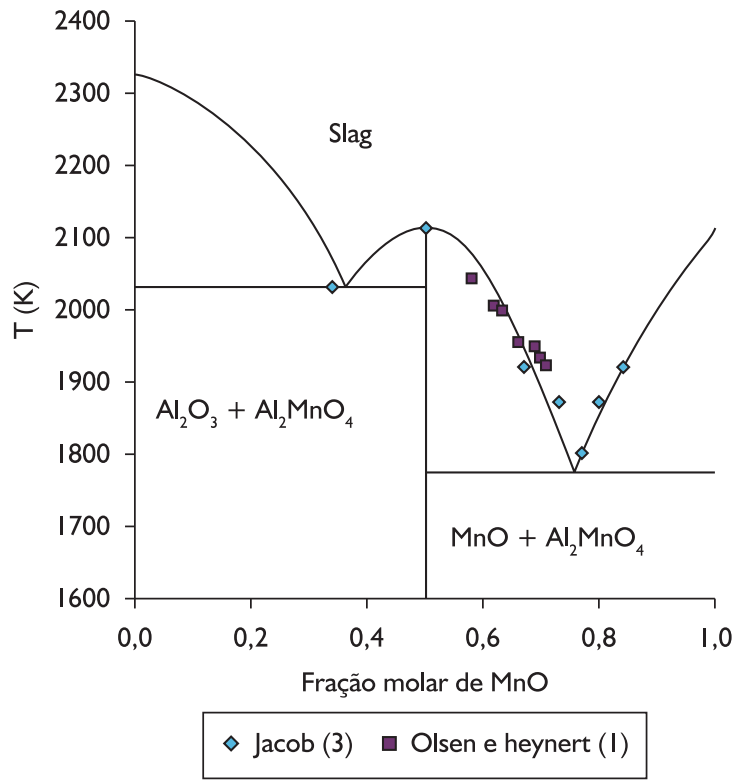

Figura 6. Diagrama de fases recalculado para o sistema $\mathrm{Al}_{2} \mathrm{O}_{3}-\mathrm{MnO}$.

Tabela 2. Propriedades invariantes calculadas com novo modelo de energia de Gibbs para o óxido $\mathrm{Al}_{2} \mathrm{MnO}_{4}$

\begin{tabular}{|c|c|c|}
\hline Reação invariante & Valores experimentais & Valores calculados \\
\hline Escória $+\mathrm{Al}_{2} \mathrm{O}_{3}+\mathrm{Al}_{2} \mathrm{MnO}_{4}$ & $\begin{array}{c}T=2.030 \pm 15 \mathrm{~K} \\
X(\mathrm{MnO})=0,34 \pm 0,02\end{array}$ & $\begin{array}{c}T=2.033 \mathrm{~K} \\
X(\mathrm{MnO})=0,27\end{array}$ \\
\hline Escória $+\mathrm{Al}_{2} \mathrm{O}_{3}$ & $T=2.114 \pm 15 \mathrm{~K}$ & $T=2.116 \mathrm{~K}$ \\
\hline Escória $+\mathrm{MnO}+\mathrm{Al}_{2} \mathrm{MnO}_{4}$ & $\begin{array}{c}T=1.802 \pm 15 \mathrm{~K} \\
X(\mathrm{MnO})=0,77 \pm 0,02\end{array}$ & $\begin{array}{c}T=1.777 \mathrm{~K} \\
X(\mathrm{MnO})=0,76\end{array}$ \\
\hline
\end{tabular}

(desordem catiônica), fenômeno este já conhecido dentre outros óxidos que também cristalizam na forma de um espinélio. (II) A possibilidade de desordem catiônica não foi considerada na construção do modelo representado pela Equação 3. Neste caso deve-se empregar o formalismo da energia composta, ${ }^{(12)}$ que utiliza a energia de Gibbs da forma estequiométrica como parte da função de referência. A função resultante apresenta dependência térmica não linear, de forma que para uma dada composição, a diferença entre o valor real e o valor calculado somente com a proposta estequiométrica não pode ser descrita pela simples adição de um termo na forma $A+B * T$.

Os parâmetros estimados (Tabela 3) apresentam magnitude significativamente inferior, quando comparados aos valores obtidos no ajuste preliminar considerando a hipótese de fusão congruente (tópico 2). A correção entálpica introduzida na energia de Gibbs do óxido $\mathrm{Al}_{2} \mathrm{MnO}_{4}$ obtida com o novo modelo de energia de Gibbs da referida fase é uma ordem de grandeza inferior ao valor estimado no ajuste do tópico (2). Outro fato notável diz respeito à concordância em nível quantitativo entre valores calculados e experimentais para os pontos da linha líquidus, dados de atividade química e valores dos patamares de isoatividade $\mathrm{Al}_{2} \mathrm{MnO}_{4}$ - escória. Jacob ${ }^{(3)}$ sugere que a desordem catiônica presente no óxido
Tabela 3. Parâmetros ajustados com o novo modelo de $G$ para o óxido $\mathrm{Al}_{2} \mathrm{MnO}_{4}$

\begin{tabular}{ccc}
\hline $\begin{array}{c}E_{\mathrm{Mn}, \mathbf{A l}} \\
(\mathrm{J} / \mathbf{m o l})\end{array}$ & $\begin{array}{c}W_{\mathrm{Mn}, \mathbf{A l}} \\
(\mathrm{J} / \mathbf{m o l})\end{array}$ & $\begin{array}{c}\text { Correção } \\
\mathbf{G}_{\mathrm{Al}_{2} \mathrm{MnO}_{4}}(\mathrm{~J} / \mathbf{m o l})\end{array}$ \\
\hline$-18 \mid 15+18347 . \mathrm{X}_{\mathrm{Al}_{2} \mathrm{O}_{3}}$ & $-33399-29908 . \mathrm{X}_{\mathrm{Al}_{2} \mathrm{O}_{3}}$ & +4357 \\
\hline
\end{tabular}

$\mathrm{Al}_{2} \mathrm{MnO}_{4}$ não deve influenciar os mencionados equilíbrios, tampouco $\circ$ ponto de fusão congruente, onde a fração molar de $\mathrm{MnO}$ na fase escória deve necessariamente apresentar magnitude igual a 0,5. A temperatura de fusão congruente calculada é de fato idêntica ao valor experimental. Cenário distinto é sugerido por Jacob ${ }^{(3)}$ no que diz respeito aos equilíbrios eutéticos. Nestas condições o emprego de um modelo estequiométrico para a energia de Gibbs do óxido $\mathrm{Al}_{2} \mathrm{MnO}_{4}$ deve gerar desvios mensuráveis em relação aos valores experimentais. Realmente, apenas para esses pontos são observados desvios superiores ao erro experimental. Este tipo de comportamento é consistente com a observação de que a desordem catiônica não foi incorporada na formulação do modelo $G$ aqui proposto Equação 3, indicando que este se encontra próximo do esperado para o óxido $\mathrm{A}_{2} \mathrm{IMnO}_{4}$, enquanto descrito como uma fase estequiométrica. 

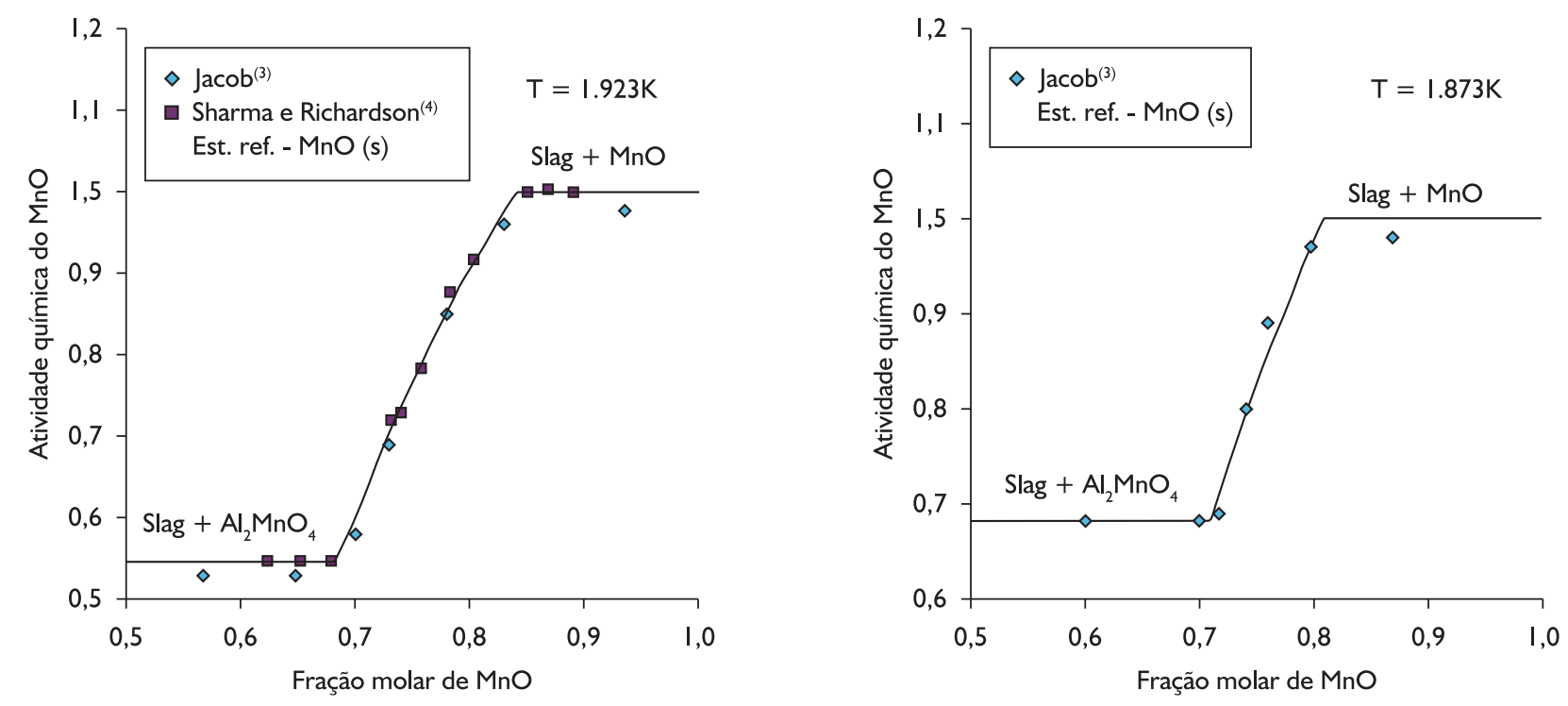

Figura 7. Atividade química do óxido $\mathrm{MnO}$ em I.923K e I.873K - fusão congruente.

\section{CONCLUSÕES}

No presente trabalho é proposto um novo modelo de energia de Gibbs para o óxido $\mathrm{Al}_{2} \mathrm{MnO}_{4}$, em sua forma estequiométrica. $O$ modelo é fundamentado em dados experimentais recentes de capacidade calorífica a pressão constante. Os resultados obtidos indicam que o novo modelo permite uma melhor descrição das propriedades termodinâmicas do sistema $\mathrm{Al}_{2} \mathrm{O}_{3}-\mathrm{MnO}$, estando, portanto, mais próximo da realidade física do óxido em questão. Este modelo pode ser futuramente utilizado na construção de uma proposta mais abrangente, fundamentada no formalismo da energia composta que incorpore em sua formulação a possibilidade de desordem catiônica.

\section{Agradecimentos}

Os autores agradecem ao CNPq. Rogério Navarro Correia de Siqueira agradece a bolsa da CAPES e a bolsa FAPERJ Nota 10.

\section{REFERÊNCIAS}

I OLSEN, W., HEYNERT, G. Die Reaktionen zwishen Eisen - mangan Schmelzen und den Schmelzen ihre Aluminate. Archiv für das Eissenhutenwessen, v. 26, n. 10, p. 567-75, Oct. 1955.

2 NOVOKAHATISKI, A. N. et al. Equilibrium diagram of the $\mathrm{Al}_{2} \mathrm{O}_{3}$ (Corundum) - MnO System. Russian Journal of Inorganic Chemistry, v. II, n. 2, p. 23I-2, Feb. 1966.

3 JACOB, K. T. Revision of the thermodynamic data on $\mathrm{Al}_{2} \mathrm{O}_{3}-\mathrm{MnO}$ melts. Canadian Metallurgical Quarterly, v. 20, n. I, p. 89-92, June 1981.

4 SHARMA, R. A.; RICHARDSON, F. D. Activities of manganese oxide, sulfide capacities and activity coeficients in aluminate and silicate melts. Transactions of the Metallurgical Society AIME, v. 233, p. 1586-1592, Ago. 1965.

5 JUNG, I. et al. Thermodynamic evaluation and optimization of the MnO-Al2O3 and $\mathrm{MnO}-\mathrm{Al}_{2} \mathrm{O}_{3}-\mathrm{SiO}_{2}$ systems, and applications to inclusion engineering. Metalurgical and Materials Transactions B, v. 35, n. 2, p. 258-68, Apr. 2004.

6 NAVARRO, R. C. S. Investigação do sistema $\mathrm{Al}_{2} \mathrm{O}_{3}-\mathrm{MnO}$ : propriedades termodinâmicas do óxido $\mathrm{Al}_{2} \mathrm{MnO}_{4}$. 2009. I I I p. Tese (Doutorado em Engenharia de Materiais) - Instituto de Engenharia de Materiais da Pontifícia Universidade Católica do Rio de Janeiro, Rio de Janeiro, 2009.

7 HACK, K. The SGTE Casebook: thermodynamics at work. Cambridge: Wood Head, 2009.

8 SOUNDERS, N.; MIODOVINKI, A. P. Calphad (Calculation of phase diagrams): a comprehensive guide. Cambridge: Pergamon, 1998. 
9 KAPOOR, M. L.; FROHBERG M, G. Theoretical treatment of activities in silicate melts. Chemical metallurgy of iron and steel. In: International Symposium on Metallurgical Chemistry - applications in ferrous metallurgy, 1973, Berlin, Germany. Chemical metallurgy of iron and steel: proceedings. London: Iron and Steel Institute, 1973. p. 17-22.

10 BERMANN, R. G.; BROWN, T. H. Heat capacity of minerals in the system $\mathrm{Na}_{2} \mathrm{O}-\mathrm{K}_{2} \mathrm{O}-\mathrm{CaO}-\mathrm{MgO}-\mathrm{FeO}-\mathrm{Fe}_{2} \mathrm{O}_{3}-\mathrm{Al}_{2} \mathrm{O}_{3}-$ $\mathrm{SiO}_{2}-\mathrm{TiO}_{2}-\mathrm{H}_{2} \mathrm{O}$ : representation, estimation, and high temperature extrapolation. Contributions to Mineralogy and Petrology, v. 89, n. 2-3, p. 169-83, Dec. 1985. http://dx.doi.org//0.1007/BF0037945 I

I I JOHNATAN, A. B. et al. Predicting lattice parameter as a function of cationic disorder in the spinel $\mathrm{MgAl}_{2} \mathrm{O}_{4}$. Journal of Physics: Condensed Matter, v. 17, p. 762I-3I, Nov. 2005. http://dx.doi.org/I0.1088/0953-8984/I 7/48/0I4

12 HILLERT, M. The compound energy formalism. Journal of Alloy and Compounds, v. 320, n. 2, p. 16I-76, May 2001. http://dx.doi.org/10.1016/50925-8388(00)0148I-X

Recebido: 6/10/2010

Aceito: 18/03/2011 